\title{
MIR210 Pre-miRNA
}

National Cancer Institute

\section{Source}

National Cancer Institute. MIR210 Pre-miRNA. NCI Thesaurus. Code C82808.

MIR210 is an oligoribonucleotide that is encoded by the human MIR210 gene and has a role in the regulation of gene expression. 